\title{
An Uncertain Future
}

\section{Geoff Goodfellow}

I was sitting in my car opposite

the Adelaide Magistrates Court

waiting on a change of lights

when i first saw her

she was in her early twenties

had on a black sleeveless top

$\&$ a denim mini skirt

her arms \& legs were heavily

tanned \& she wore strappy sandals

her hair was bottle blonde-

$\&$ as she crossed in front of me

blowing out a stream of blue

cigarette smoke

i noticed her black roots

complimented her chipped \& broken

front teeth

she was at least seven months pregnant

the lights changed

i moved off slowly-

into my own uncertain future. 